\title{
TUMOURS OF THE PARIETAL AND OCCIPITAL LOBES.
}

\author{
By A. DICKSON WRIGHT, M.S., F.R.C.S. \\ (Assistant Surgeon, St. Mary's Hospital; Surgeon, Maida Vale Hospital.)
}

Tumours of the parietal and occipital lobes by their situation in functioning parts of the brain very early produce signs and symptoms of localising value. Hence a reasonable forecast of the site and even the type of tumour may be made from the clinical examination and history of these cases.

Occipital tumours reveal themselves by their interference with the visual fields and the loss of field is naturally in the form of a homonymous hemianopia, at first partial, but as a rule becoming complete very rapidly; more rapidly than in the case of hemianopia from the downwards pressure of a parietal or the upward pressure of a temporal tumour upon the optic radiation. In these latter cases the hemianopia has a tendency to be at first quadrantic with the lower quadrants first affected in parietal cases and the upper quadrants in temporal cases. Of great interest in the homonymous hemianopia due to occipital tumours is the sparing of the macular area across the midline, this occurring even when the occipital lobe is completely destroyed by the tumour or completely removed by operation. There has been much speculation as to the reason for this and it is suggested that a decussation of some of the macular fibres occurs further forward and the macular region is thus bilaterally represented. The preservation of the whole macular area is often of great benefit to the patient, making his homonymous hemianopia much less of an infliction.

As an occipital tumour grows, it may press upon the cerebellum and certain? cerebellar symptoms may appear, such as ataxia and nystagmus. Again, in "spreading" forward, the actual infiltration of the tumour or the peri-tumoral œdema or the cerrebral dislocation produced by the mass of the tumour may lead to the development of parietal signs, such as astereognosis and hemiparesis, by pressure upon the Rolandic gyri. On the left side involvement of the reading centre results in word blindness (alexia).

Epileptiform convulsions occur in about 40 per cent. of intracranial tumours and the aura in occipital tumours may be of a visual type occurring in the affected halves of the visual field. It is said that when the tumour is actually in the visual cortex, the aura are crude, consisting of balls or flashes of white or coloured light, whereas when the lesion affects the visual tracts, the aura are of more complicated kind, very definite visual images being seen as the fit commences. When forward extension of the tumour occurs, the fits may be of a sensory or motor Jacksonian type. There is no special type of tumour with an affinity for the occipital lobes. Meningiomata are rarely found as far back as this region, so unfortunately many of the tumours here are glial.

The usual route for ventricular puncture traverses the occipital lobe so that the tumour or its associated cyst may be struck during this procedure. If a cyst is struck, it is wise to empty it as far as possible, replace with air so as to obtain an accurate X-ray of its extent and possibly the site of a mural nodule, so that the operation can be carefully planned. An even more accurate topography of the cyst can be delineated by thorotrast filling and this procedure does not produce the dangerous thorotrast reactions as when this substance is introduced into the ventricle. Again, a sample of the tumour may be obtained with the brain needle 
or a special biopsy punch and, if malignant spongioblastoma cells are found with certainty, then the patient may be left to his inevitable fate and spared the trouble, expense and disappointment of a fruitless intervention.

The exposure of the occipital lobe is usually done through a flap based on the temporal muscle, but the flap may be based upon the occipital ridge, this being slightly easier to make with the patient lying upon his face which is the best operating position for this part of the brain; but if this latter flap is used, it is more difficult to leave a decompression.

The operation of occipital lobectomy should be well considered especially in cases of oligodendrogliomatous tumours and non-cystic astrocytomata with indefinite outlines. The incision in the cortex on the convexity of the brain can be made further forward than the ordinary anatomical boundary of the occipital lobe, because of the displacement produced by the large tumour.

Parietal tumours form a much larger group than the occipital tumours. Their symptoms obtrude more upon the patient's perception because although a homonymous hemianopia may be unnoticed for quite a long time by a slightly obfuscated patient, the same does not apply to a hemiplegia or an aphasia which are very obtrusive symptoms. It is of some historical interest that the first brain tumour ever operated upon was in this region over fifty years ago. The tumour was localised as a result of the then recent discoveries in cerebral localisation by Sir David Ferrer. The operation was performed by Lord Lister's nephew, Sir Rickman Godlee, and the patient, a Scottish farmer, died three weeks later with a large cerebral fungus, as the tumour was unfortunately sub-cortical and of an infiltrating unencapsulated type.

The first signs of a parietal tumour are often in the form of epileptiform fits with a localising aura such as peculiar sensations in one limb or half of the face or even half of the body. Again it may be noticed that the first movements are in one limb or the half of the body. An examination of the patient immediately after the fit may disclose a clear and definite hemiplegia, which may disappear after a few hours. The fit is sometimes of the Jacksonian type, the movements starting in one-small muscle group and extending slowly to a varying extent over that side of the body. It often happens that in a fit of this kind the patient is conscious throughout the whole fit and watches the march of the convulsions; in the more severe cases consciousness is lost. After such a fit, the hemiplegia is always much more marked for several hours or even days.

Aphasia is an important localising sign in left-sided parietal tumour. It starts with a hesitation in starting a sentence (aporia) and later words are dropped and the speech may be confined to a few monosyllables, such as "yes" or "no", and then finally the patient may confine himself to some meaningless sound which he repeats monotonously (perseveration) or may be completely mute. Very interesting are cases where the patient, aphasic in his parent tongue, is nevertheless able to converse in a foreign language. A historical case of this is that of Dr. Johnson who in his last days could speak no word of English, but could abuse his doctors roundly in Latin!

In posterior parietal lesions on the left side, word blindness (alexia) may result from involvement of the angular gyrus. Sometimes in these cases one may have the amazing condition of a patient able to write from dictation and yet unable to read what he has just written. In left-handed patients the speech centres are on the right side of the brain, but it must also be remembered that persons who start 
life left-handed and are forced to become right-handed and those belonging to lefthanded families may have speech centres on the wrong side.

Sensory losses due to involvement of the post-Rolandic gyrus strangely do not take the form of an absolute hemianæsthesia; it is very rare for any intracranial condition to produce this condition. It is more common to lose the sensory perception of form so that the patient cannot recognise well-known objects (pencil, coin, etc.) placed in the hand; this condition is called astereognosis. It may also be caused by high cervical cord tumours and tumours in the foramen magnum, but when it is confined strictly to one hand, a lesion involving the sensory cortex is very probable.

The involvement of the visual pathway has already been mentioned and the homonymous field defect is often quadrantic at first, affecting the lower quadrants as a rule.

The parietal lobes are frequently the site of meningiomas, these tumours when occurring on the convexity of the brain having a very special affinity for the motor areas. The presence of such a tumour may be inferred by the slow march of motor symptoms and the great frequency of Jacksonian attacks in these slow-growing tumours. Some X-ray signs also may point to a meningioma, such as increase of the diploic venous channels in the bone over the tumours and increase in the size of the meningeal vessels and the aperture of the foramen spinosum. Bone changes in the form of rarefaction, erosion or hyperostosis may also appear over this tumour and indicate the correct pathological diagnosis and, finally, a spicule of bone from the inner table may indicate the root of the tumour or an area of calcification at the root of the tumour may differentiate it from the glioma with its diffuse patchy calcification.

One special type of meningioma of great practical importance may produce a very typical syndrome, that is the parasagittal meningioma which, as its name implies, grows between the hemisphere and the falx cerebri. Here it will naturally affect the leg centres first and the motor effects will spread slowly upwards in the body as the tumour grows downwards in the brain. The foreknowledge of this type of tumour is of great importance in planning the operative attack.

The malignant gliomata seem to have a special predilection for the parietal region and the picture of a patient between the ages of 40 and 60 with a rapidly progressing hemiplegia with epileptiform seizures starting from the hemiplegic side is a very ominous one. On exposure of such a lesion the gyri are seen to be much expanded and flattened and possibly some tumour will be showing upon the surface. Needling of the depths of the tumour discloses cystic and hæmorrhagic areas.

The results of the operative disturbance of these tumours are generally deplorable. A partial hemiplegia or aphasia becomes total and if a decompression is provided by leaving the bone flap unwired, then a huge hernia may develop and the patient, unable to die from increased intracranial pressure, lives on and no one is grateful for perpetuating such a miserable existence.

It is therefore very wise to endeavour to diagnose these malignant gliomata before operation and there are three ways in which this may be done.

I. The short history and the rapid progress to be observed during a short period of observation are of great value, but it must be remembered that sometimes a benign condition, such as a cystic astrocytoma or an abscess, after a quiet almost unnoticed existence will suddenly, as a result of hæmorrhage or œedema, produce a 
rapidly progressive syndrome. In other cases the dislocation produced by a slowgrowing tumour will cause some ventricular blockage and the development of a hydrocephalus causes rapid progress. It has also been suggested by Oljenick that when the wall of a cyst comes into contact with the lining of the ventricle osmosis occurs and produces a rapid increase in the size of the cyst. It is seen, therefore, that the history, even when most carefully studied, could lead to a very serious mistake. It is wise in a case of the slightest doubt to go further in the diagnosis.

2. Biopsy of the tumour can be made by a brain needle or a special punch such as that designed by Harvey Jackson, using a small trephine hole for the purpose.

3. Arteriography reveals the highly vascular spongioblastoma to resemble an arterio-venous aneurism with sinusoids connecting the arteries and veins and giving a picture which is in most cases recognisable to one well acquainted with arteriographic appearance.

When a tumour of this highly malignant type is diagnosed indubitably, it is generally best to abandon hope. Occasionally an exploration may be done on the right side and a large amount of tumour and adjoining brain removed so as to leave a large cavity. The bone flap is then wired securely back so that no decompression is provided. The patient will then be well for six months and able to put his affairs in order and then, when the inevitable recurrence comes, a rapid exitus eventuates.

Arterio-venous aneurisms of congenital origin are very prone to affect the parietal region and they may be suspected when there is a very long history of epileptiform convulsions and signs of cerebral tumour without the rate of progress of tumour growth that one would expect even in a most benign meningioma. These tumours consist of a plexus of gigantic blood vessels with often a solid core of angiomatous tumour. A murmur may often be heard through the skull and failure to auscultate may be very much regretted in such a case because exploration is fraught with very great danger in these cases and is frequently of very little value. The best treatment consists in ligation of the carotid artery and treatment of the tumour area by radium or X-rays.

$X$-ray appearances of parietal tumours are very characteristic. In plain X-rays the pineal shadow will be almost invariably displaced to the unaffected side. The ventriculograms will show displacement of the ventricular system to the opposite side, perhaps some inclination of the third ventricle from the vertical and sometimes the temporal horn is prevented from filling. In small tumours the deformity of the body may be small but very important, especially in parasagittal tumours, when a small depression in the roof of the body of the ventricle may be the only sign. 